\title{
LA VOZ DEL ALUMNADO: SU SILENCIO Y LA CULTURA PROFESIONALISTA
}

(THE STUDENT VOICE: HIS SILENCE AND THE PROFESSIONAL CULTURE)

Paula Escobedo Peiro

Auxiliadora Sales Ciges

Joan Traver Martí

Universitat Jaume I

DOI: 10.5944/educXX1.19045

Cómo referenciar este artículo/How to reference this article:

Escobedo Peiro, P.; Sales Ciges, A. y Traver Martí, J. (2017). La voz del alumnado: Su silencio y la cultura profesionalista. Educación XX1, 20(2), 299-318, doi: 10.5944/educXX1.19045

Escobedo Peiro, P.; Sales Ciges, A. \& Traver Martí, J. (2017). La voz del alumnado: Su silencio y la cultura profesionalista. [The student voice: His silence and the professional culture]. Educación XX1, 20(2), 299-318, doi: 10.5944/educXX1.19045

\section{RESUMEN}

El artículo que presentamos se centra en el estudio realizado en una escuela de Educación Infantil y Primaria. El objetivo de dicho estudio es conocer la voz del alumnado en el centro, y sobre todo entender por qué en una escuela que intenta transformarse para ser más intercultural e inclusiva, la voz del alumnado queda silenciada. Metodológicamente se trata de un estudio de caso, en el que mediante grupos de discusión, entrevistas y observación participante tratamos de comprender las perspectivas de los participantes en profundidad. Los principales resultados apuntan a la cultura profesionalista de los maestros y las maestras, como un aspecto que influye negativamente en la participación de las familias y del alumnado. Además, el hecho de escuchar la voz del alumnado, nos permite tener una visión más completa del centro y conocer algunas de las contradicciones que se dan en la práctica. Como conclusiones, destacamos la importancia de rescatar la voz del alumnado y fomentar su participación activa en el centro, no solo para crear una escuela más participativa y democrática, sino también para obtener una mirada más completa de la institución.

\section{PALABRAS CLAVE}

Percepción del alumnado; cultura profesionalista; modelo intercultural inclusivo. 


\section{ABSTRACT}

The present article focuses on a study in a childhood and Primary Education centre. The objective of this study is to learn the student voice in the centre, and understand why, in a school that tries to transform to become more intercultural and inclusive, the student voice is silenced. Methodologically, this is a case study in which, through focus groups, interviews and participant observation we try to understand the perspectives of the participants in depth. The main results include the professional culture of teachers as an aspect that negatively influences the participation of families and students. Also, the fact of listening to the voice of the students gives us a more complete view of the centre and shows some of the contradictions that exist in the practice. As conclusions, we highlight the importance of rescuing the student voice and encouraging their active participation in the centre, not only to create a more participatory and democratic school, but also to get a more complete look of the institution.

\section{KEYWORDS}

Student Teacher Evaluation; perception; school culture.

\section{INTRODUCCIÓN}

El presente artículo trata de dar a conocer la situación del alumnado de un centro educativo que desde el año 2007 intenta transformar la institución hacia un modelo intercultural e inclusivo. Se trata de un CAES (Centro de Acción Educativa Singular) ${ }^{1}$ situado en una ciudad de la provincia de Valencia. Esta escuela atiende a alumnado de Educación Infantil y Primaria y además, tiene un aula autorizada como aula especial de primer ciclo de la ESO para alumnos/as con riesgo de abandono de la escolaridad. La plantilla del profesorado es de 19 personas y hay 140 alumnos, de los que un $89 \%$ pertenecen a familias de etnia gitana y el $11 \%$ restante son personas inmigrantes y/o en una situación de desventaja social.

En este estudio, que realizamos desde el grupo MEICRI ${ }^{2}$, pretendemos desvelar por qué existe un silenciamiento en la voz del alumnado, siendo una escuela que pretende transformarse hacia un modelo más participativo y democrático. La finalidad que perseguimos es visibilizar la voz del alumnado de esta escuela así como resaltar la importancia de aquello que ellos nos cuentan.

La metodología utilizada responde al estudio de caso. En concreto, nos acercamos al objeto de estudio utilizando técnicas y estrategias que nos per- 
miten recabar datos cualitativos. Estas técnicas son los grupos de discusión, las entrevistas y la observación participante.

Como principales aportes, destacamos la importancia de establecer una cultura participativa y democrática en los centros, que supere un profesionalismo de la acción educativa que recaiga únicamente en los y las docentes.

\section{LA VOZ DEL ALUMNADO COMO AGENTE DE CAMBIO}

Respecto al concepto "voz del alumnado», nos basamos en Susinos (2012) al definirla como un término bastante común en otros países, principalmente anglosajones, para referirse a «todas aquellas iniciativas que emprenden las escuelas y que están dirigidas a aumentar el protagonismo del alumnado en la toma de decisiones sobre el diseño, la gestión y la evaluación de cualquier aspecto de la vida escolar» (p. 16). En los últimos años las experiencias que visibilizan la voz del alumnado en el ámbito nacional han sido múltiples (Traver, Sales y Moliner, 2010; Rojas, Haya y Lázaro, 2012; Susinos y Ceballos, 2012; Susinos, 2013). En esta misma línea, con el presente artículo pretendemos abrir otra ventana que nos permita ver cómo es esa voz y entender algunas de las contradicciones que se encuentran en la práctica.

La primera tesis que defendemos es la necesidad de tener en cuenta la voz del alumnado para aquellos centros que pretenden transformar sus estructuras hacia modelos de participación horizontal, basados en enfoques democráticos. La segunda idea que mostramos es que, independientemente del modelo educativo por el que se rija la escuela es importante tener en cuenta la voz del alumnado, para favorecer la creación de escuelas más democráticas y participativas. Finalmente, defendemos que la mirada del alumnado sobre las acciones que se realizan en la escuela aporta una visión más completa y compleja sobre la realidad vivida en los centros. Aunque no siempre es fácil visibilizar su voz es importante el papel del profesorado para conseguirlo. En contraposición a este planteamiento, podemos hallar posicionamientos de los y las profesionales de la educación que, situados en una cultura profesionalista, resultan ser un impedimento para conseguir participaciones más auténticas y reales de otros agentes educativos.

Empezaremos situando la voz del alumnado en el caso que explicamos, una escuela que lleva dos años realizando acciones para ser más intercultural e inclusiva. Desde este modelo, la participación democrática de todos los agentes es una premisa. Es decir, se presupone la horizontalidad y se realizan acciones que incluyan la participación de todas las personas. Por 
eso, para que un centro sea inclusivo, tanto la escuela como las aulas tienen que ser entendidas desde la perspectiva de los diferentes participantes «incluidos los propios niños» (Ainscow, 2002, p. 70).

En segundo lugar, pensamos que es importante tener en cuenta la voz del alumnado, independientemente de la filosofía escolar del centro. Como hemos comentado, en escuelas que ya se han planteado el reto de transformarse hacia la inclusión y la interculturalidad, la participación de los distintos agentes de la comunidad tiene que ser un hecho. Sin embargo, en todas las escuelas podremos ir encontrando cambios en la medida en la que se abra la participación del alumnado en la escuela (Márquez y Sandoval, 2016). Como destaca en su tesis doctoral Bucknall (2009), aunque a lo largo de los años ha habido cambios con respecto a la voz de los y las estudiantes, este aspecto sigue siendo problemático. La autora plantea que ha cambiado el estatus del alumnado en algunos centros, pero puede que su opinión siga sin ser escuchada de manera significativa. A dicha cuestión, Susinos y Ceballos (2012) responden que se puede ir avanzando hacia modelos de voz del alumnado más comprometidos con una participación real, en la medida en que haya un mayor interés por conocer su punto de vista y se propicie un mayor debate y autonomía.

En tercer lugar, cabe destacar que aunque la voz del alumnado es importante para conseguir escuelas más abiertas a la comunidad, en muchas ocasiones sus voces quedan silenciadas por una cultura profesionalista. Entendemos este concepto en el sentido que señala Imbernon (2007), desde una concepción neoliberalista de la profesión docente que se atribuye al término profesionalismo. Se trata de una forma de entender la cultura profesional del profesorado que este autor califica de reduccionista y que impide su desarrollo profesional como colectivo, tanto a nivel social como educativo. Como Imbernon alerta, este posicionamiento docente puede llevar al profesorado a adoptar actitudes y comportamientos prepotentes, basados en pretensiones de poder y anclados en un saber técnico, específico patrimonio del propio colectivo. Desde una perspectiva profesionalista, para Guerrero (2007) es interesante analizar la posición de autoridad que los y las docentes ocupan en la escuela, así como su relación con los demás sectores de la comunidad educativa, en especial padres y alumnado. Para este autor, siguiendo los trabajos sociológicos clásicos sobre el profesorado de autores como Becker (1953) y Waller (1932), esta forma de entender la docencia y el estatus que asumen como profesionales especializados, hace que perciban a los demás sectores educativos como intrusos e ignorantes en relación a lo que sucede en las aulas.

Además, la cultura profesionalista basa las relaciones de poder en el estatus que da a los docentes, u otros profesionales de la educación, dispo- 
ner de conocimientos especializados, y en la legitimidad que estos le dan para tomar decisiones sin sufrir interferencias externas. Si se entiende la profesionalidad del profesorado desde un marco de relaciones sociales justas y democráticas, su reconocimiento social está más ligado a sus capacidades para desarrollar un liderazgo inclusivo y sostenible (Gale y Densmore, 2007; Hargreaves y Fink, 2008). Por eso, destacamos que la transformación de una escuela hacia modelos de participación democrática no supone únicamente el cambio en algunas prácticas de aula o en acciones concretas del centro, sino un cambio en la mirada docente.

Como dice Echeíta (2011) los y las docentes «ya no son simplemente conductores de conocimientos, sino guías de los niños» (p.124). Este cambio en el posicionamiento de maestras y maestros, no tiene que ver únicamente con aspectos procedimentales, sino que como señalan Rojas y otros (2012) «conlleva la manifestación de actitudes de respeto y escucha auténtica acerca de los asuntos que los alumnos plantean» (p. 93).

Es precisamente este cambio de mirada el que puede ser conflictivo, pues no se trata de realizar unas determinadas acciones, sino de un cambio de paradigma. Cambio que muchas veces el docente entiende y se plantea por sus propios valores, pero que en otras ocasiones pueden suponerle un conflicto interno. Pues los cambios en la cultura exigen entender las innovaciones como una actividad no solo individual sino también cooperativa, replanteando el concepto de liderazgo y profesionalización docente (Marcelo y Estebaranz, 1999). Se trata pues de transformar la cultura docente profesionalista en una cultura docente colaborativa, que cuente con todos los miembros del centro. Sería, en palabras de Imbernon (2007), pasar a una cultura docente basada en un concepto de la profesión docente «más social, más dinámico, más complejo [...] y multidimensional, en el que el proceso de profesionalización se forja en un contexto de democracia, de auge de valores contradictorios entre los individuos y el progreso social» (p. 14).

Finalmente, Susinos y Ceballos (2012) destacan que el estadio último donde la voz del alumnado está más presente debería situarse en el horizonte de un proyecto inclusivo, que Fielding (2011) denomina «aprendizaje intergeneracional como forma de vida» (p. 32). En la que el profesorado y alumnado se sitúan como aprendices e investigadores. Se trata de un aprendizaje compartido entre jóvenes y adultos, alumnado, familias, profesorado y vecindario. Siempre y cuando este proceso no se viva como un hecho puntual sino como la forma habitual de gestionar el centro. Como explican las autoras, que dicha manera de actuar se refiera tanto a lo curricular como a lo organizativo, y «que adultos y jóvenes aprendan unos de otros durante el proceso de trabajo compartido y en 
los espacios de diálogo con una finalidad deliberativa sincera» (Susinos y Ceballos, 2012, p. 29). En definitiva, sería la manera de vivir la democracia en la escuela.

Sería pues, la oportunidad para el alumnado de experimentarse como agente de cambio y de aprender que sus acciones pueden influir sobre la realidad (Rojas y otros, 2012). Intención que Santos Guerra (2010) destaca como una de las principales funciones de la escuela.

\section{DISEÑO DE LA INVESTIGACIÓN}

En este artículo tratamos de explicar una parte del estudio realizado en una escuela en transformación hacia el modelo intercultural inclusivo. En dicho estudio, nuestro objetivo era desvelar las dificultades del proceso de transformación, especialmente comprender los cambios que se iban produciendo en la cultura escolar del centro y en su enfoque educativo para abordar la diversidad. Puesto que, habiéndose planteado dicho cambio dos años atrás, tenía dificultades para realizar acciones basadas en la participación democrática y en la inclusión.

De todos los elementos que nos ayudan a explicar las dificultades de esta escuela a lo largo del proceso de transformación, en el presente artículo nos basamos en conocer cómo es la voz del alumnado, y poder aproximarnos a las causas de su posible silenciamiento. Nuestra participación como asesores en el proyecto de construcción de una escuela intercultural inclusiva, nos alerta sobre cierta contradicción entre el discurso y las prácticas educativas que continúan invisibilizando al alumnado del centro escolar. El problema de investigación trata de comprender las razones que explican por qué en una escuela en proceso de transformación hacia la inclusión y la interculturalidad, la voz del alumnado queda silenciada. La pregunta de investigación que nos hacemos al respecto es ¿Por qué la voz del alumnado queda silenciada en esta escuela?

Por las características de nuestro estudio nos acercamos a la investigación cualitativa, ya que pretendemos comprender una realidad en profundidad y responder a nuestro problema y preguntas de investigación desde datos cualitativos.

Metodológicamente se trata de un estudio de caso. Nuestra intención es estudiar la particularidad y complejidad de esta escuela en concreto, por las características contradictorias que presenta, para llegar a comprender su realidad (Stake, 2007). 


\section{Trabajo de campo}

Nuestra entrada en el campo se inició en enero de 2010. Al mismo tiempo que nosotros realizamos la investigación, en el centro escolar se estaba realizando un taller sobre resolución de conflictos destinado al alumnado, profesorado y familias. Para preparar el taller el profesorado decidió indagar en el conocimiento que tenían las familias, el alumnado y también el profesorado sobre el tema. Para ello realizaron grupos de discusión en los que preguntaron a los distintos colectivos (familias, alumnado y profesorado) sobre los conflictos del centro. Esta parte que se utilizó para el taller formó también parte de nuestra investigación, como un aspecto más sobre el que indagar para conocer la realidad del centro. Además, realizamos entrevistas con el profesorado y el alumnado y asistimos como observadores participantes a las distintas sesiones realizadas. El trabajo de campo terminó en junio de 2010, en una última sesión conjunta con el profesorado para discutir los resultados del estudio (día 04.06.10).

\section{Técnicas y estrategias de recogida de datos}

En los grupos de discusión, el profesorado fue quien delimitó los participantes. Se realizaron dos grupos de discusión con el alumnado, uno con las familias y otro con el profesorado. En los grupos del estudiantado, en uno participaron cinco alumnos y tres alumnas de sexto de Primaria y del aula de primer ciclo de la ESO. En el otro, cinco alumnos y tres alumnas de segundo ciclo y quinto de Primaria. De esta manera pudimos obtener una muestra representativa del estudiantado de todo el colegio.

Respecto el grupo de discusión del profesorado, se contó con un representante de cada una de las etapas educativas y de cada ciclo de la etapa de Primaria. Además, participaron los tres miembros del equipo directivo y una persona del equipo MEICRI como observadora, un total de dos hombres y siete mujeres.

En el grupo de discusión de las familias no se realizó una preselección de los miembros. Puesto que el profesorado indicó que había poca participación de las familias en la escuela, decidieron abrir el grupo de discusión a todas aquellas familias que quisieran participar. Finalmente asistieron cinco madres y un padre.

Para establecer el protocolo de preguntas en los grupos de discusión, se tuvieron en cuenta aspectos teóricos sobre resolución de conflictos y el conocimiento que se tenía sobre el clima de convivencia en el centro. De esta forma, los temas centrales en los grupos de discusión fueron: qué con- 
flictos había en el centro; cuáles se repetían con mayor frecuencia y cuáles se consideraban más graves; cómo se actuaba ante el conflicto; las normas de convivencia del centro; la implicación de las familias en la convivencia; la mediación como un modelo de resolución del conflicto.

Para las entrevistas, realizamos un muestreo teórico que nos permitiera obtener una representación de distintos posicionamientos del profesorado del centro. Seleccionamos a tres docentes X, N e Y. Entre ellos, había una persona muy implicada con el centro; otra persona reacia al cambio y otra persona que había estado fuera de la escuela durante cuatro meses y aportaba una mirada externa, siendo conocedora de la realidad escolar. En el caso del alumnado, puesto que nuestro conocimiento sobre este era menor que el del profesorado, fue el equipo de docentes quienes hicieron la selección: un alumno con un comportamiento solidario y una actitud positiva en el centro y una alumna con una actitud menos colaboradora y un comportamiento conflictivo. Realizamos una segunda entrevista con las mismas personas, para profundizar en aquellos aspectos más relevantes sobre los que necesitábamos más información.

En este caso, las preguntas que guiaban la entrevista estaban orientadas a obtener más información sobre aspectos que habían aparecido en los grupos de discusión y sobre los que se quería indagar más. Los temas principales de la entrevista fueron el papel docente; la implicación de las familias en la escuela; los valores compartidos entre los miembros de la comunidad educativa; la resolución de conflictos; la mediación y la filosofía del centro.

Finalmente, la observación participante implicó la inmersión en la vida del centro durante el período en el que estuvimos realizando el trabajo de campo. Como observadores, mantuvimos un rol activo y una reflexión permanente sobre las situaciones y las interacciones que se daban entre las personas de la institución (Cohen y Manion, 1990). La recogida de datos mediante la observación se configuró en notas de campo, que la investigadora principal tomó en las reuniones de trabajo con el profesorado y en las sesiones del taller realizadas.

\section{Análisis de datos}

Se realizó un análisis cualitativo para poder responder al problema y a las preguntas de investigación.

En primer lugar, recogimos los datos mediante las técnicas explicadas anteriormente. Estos quedaron registrados en archivos de audio que fueron transcritos y en notas de campo. Todos los datos obtenidos se triangula- 
ron y se sistematizaron en un conjunto de categorías que iban emergiendo del propio análisis, en lugar de partir de categorías teóricas construidas desde la literatura. Para ello, necesitamos ahondar en los datos y tener un conocimiento profundo de los mismos. Leer más allá de aquello que los informantes nos aportaban y seguir indagando (observando y preguntando) para poder tener una visión clara y profunda de la cuestión. Como explican Rubin y Rubin, (1995), la construcción de la categoría consistió en agrupar por temas los conceptos emergentes en el análisis. En este proceso tratamos de establecer categorías inclusivas, que recogieron el sentido de cada uno de los conceptos agrupados. Dicho análisis fue realizándose a medida que se recogieron los datos, hasta la saturación de cada una de las categorías, es decir, hasta que los datos nuevos no aportaron más información sobre las categorías (Glaser y Strauss, 1967).

\section{RESULTADOS}

A continuación expondremos los resultados para responder a la pregunta ¿Por qué la voz del alumnado queda silenciada en esta escuela? Para ello, adjuntamos un esquema (véase Figura 1) en el que situamos las distintas categorías extraídas del análisis. Seguidamente, pasaremos a detallar cada una de ellas.

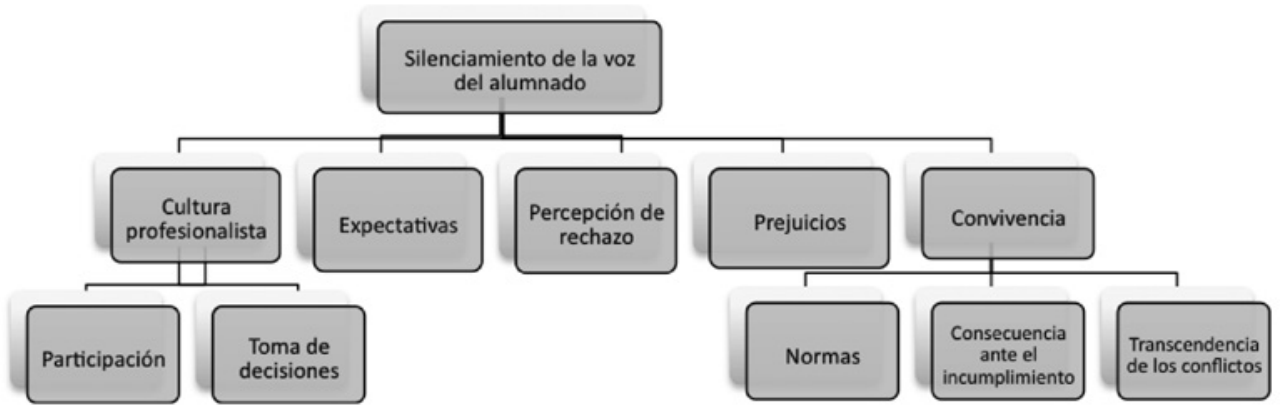

Figura 1. Silenciamiento de la voz del alumnado

A partir de los datos, entendemos que en la escuela existe una cultura profesionalista que influye en la dinámica del centro. Esta cultura es visible en las distintas sesiones que comparten los miembros del grupo de investigación junto con el profesorado. En ellas, una parte del profesorado mantiene una actitud de rechazo hacia algunas familias y el alumnado.

Respecto a la participación de las familias y el alumnado en el centro, el profesorado la limita, actuando siempre que es posible como actor único 
del quehacer escolar. Aunque podemos destacar alguno de los momentos en los que pudimos observar la intención del profesorado de abrir la participación a familiares y alumnado. Por ejemplo, en el taller de resolución de conflictos. En este, el profesorado quería abrir las sesiones de formación a familiares y estudiantado, pero finalmente el mismo profesorado decidió destinar una sesión de las cuatro, a las familias y el alumnado. Sin embargo, incluso en este caso, la formación de las familias y del estudiantado se acabó realizando aparte de la del profesorado. En definitiva, la participación no es un aspecto negociado con las familias y el alumnado, sino que es el profesorado quien decide en qué participar y en qué momentos.

"A veces, se les piden cosas de las que ellos no se sienten capaces $y$ se aturrullan, o a veces se exigen cosas que para nosotros son importantes y para ellos no. A lo mejor es que no les hemos planteado ¿En qué queréis participar? En lugar de preguntar, ¿queréis participar en esto?» (Nota de campo: docente en la sesión del 04.06.10).

Por tanto, aunque una parte del profesorado pueda explicitar que les gustaría que las familias participaran más en las acciones del centro para que sus hijos también la valoraran más (Sesión 2 del taller sobre resolución de conflictos, 05.02.10), no se establecen momentos y tiempos para que sea posible una participación activa.

La voz del alumnado nos aporta a este hecho una mirada más cercana sobre la realidad que viven las familias; en la que la participación se limita a la asistencia a las reuniones que convoca el profesorado del centro y a colaborar en la preparación de fiestas que se realizan en la escuela (Entrevista a alumno de sexto de primaria, 11.05.10).

En los grupos de discusión del alumnado de tercer ciclo de primaria y primer ciclo de la ESO (20.01.10) el estudiantado manifiesta que le gustaría también participar en más aspectos de la escuela y que sus familias también lo hicieran. Por ejemplo en la elaboración de normas de convivencia (categoría que explicaremos más adelante).

Por otra parte, no solo la participación, sino también la toma de decisiones es un aspecto clave para generar una cultura escolar más democrática y participativa. Al respecto, una parte del profesorado suele manifestar su desacuerdo en que las familias y el alumnado puedan optar a tomar decisiones que se tengan que llevar a cabo. El alumnado y las familias opinan sobre aspectos del centro, pero no llegan a tomar decisiones. Puesto que no participan en el centro, no se establecen tiempos y espacios para discutir con ellas y ellos. Por otra parte, encontramos datos que muestran que tanto las familias como el alumnado opinan y realizan propuestas al profesorado para que estas 
puedan llevarse a cabo. Por ejemplo, tras la sesión del taller de resolución de conflictos una madre se acercó a la directora pidiendo realizar un curso de mediación en la escuela. Esta propuesta fue escuchada por la directora, pero no llegó a explicarse en las sesiones junto con el resto del profesorado y tampoco se estableció una sesión para discutir el interés de la propuesta o la viabilidad de la misma con el resto de agentes del centro.

Por otro lado, la toma de decisiones sobre aspectos del currículum también es un aspecto que recae en manos del profesorado. Aunque una parte de las familias y del alumnado llega a tomar algunas decisiones en la comisión de fiestas, estas no inciden en aspectos relevantes de la organización ni desarrollo curricular del centro.

También en los datos encontramos que las bajas expectativas influyen a la hora de experimentar una apertura de la escuela hacia las familias y el alumnado.

«Los niños cómo van a tomar decisiones, y a estos padres con su cultura, no se les puede pedir más» Y seguimos teniendo una posición de maestro que somos los que realmente sabemos, los profesionales y los que tenemos que tomar decisiones. Una posición en la que cuesta abrirse. (Primera entrevista a docente Y, 30.03.10).

Además, de las bajas expectativas encontramos una distancia entre las familias, el alumnado y el profesorado.

Partimos de una escuela donde el alumnado del centro proviene de familias con una situación de desventaja social. Se trata de alumnado con problemas de vivienda, higiene, género, alimentación,... son personas faltas de todo. (Primera entrevista a docente X, 22.03.10).

Como dice el docente $\mathrm{N}$, se trata de estudiantes que a diario evidencian contextos de violencia y en algunos casos participan de ella. Opinan que el centro educativo es el puente de conexión entre el alumnado y una sociedad que quizás ni las familias ni el alumnado entienden y en la que no participan (Entrevista a docente X, 22.03.10).

La educación es la única oportunidad de que vean que hay otra manera de vivir y salir del estancamiento y de la discriminación que tienen por su etnia. (Primera entrevista a docente $N$, 30.03.10.).

Por su parte, el alumnado percibe la escuela de distintas maneras: como un lugar donde formarse para conseguir estudios superiores; donde aprender a leer, escribir, sumar y restar (competencias que consideran útiles 
en la vida cotidiana); finalmente, también como un lugar descontextualizado y alejado de sus intereses.

El alumnado nos habla sobre la utilidad de ir a la escuela y de sus expectativas de futuro. En una de las entrevistas realizadas, un alumno señala su deseo de continuar sus estudios para llegar algún día a ser maestro. Nos habla de las asignaturas que más le gustan y del tipo de trabajo que más le motiva, como el hecho de trabajar en equipo.

A veces estamos en grupos interactivos y nos dan una hoja para tres o cuatro niños, y tenemos que estar todos en grupo: uno lee, otro escribe, otro escucha, otro piensa. Y entre todos pensamos y estamos todos atentos. Y eso es trabajar en grupo y a mí me gusta mucho. (...) Aparte, que hacemos el trabajo más rápido, aprendemos rápido y aprendemos todos a la misma vez. Eso me gusta a mí. (Entrevista a alumno de sexto de primaria, 11.05.10).

En otra de las entrevistas, la alumna destaca de la escuela el aprendizaje de la lectura y la escritura, así como otros conocimientos matemáticos: sumar, restar, multiplicar y dividir. Indica que esto es necesario para poder ir a comprar o entender la información con la que se puede encontrar en el día a día. Sin embargo, está deseando terminar el colegio porque se aburre. Cree que cuando termine el colegio sus padres la apuntarán a hacer un curso de formación; al instituto decidieron que era mejor que no fuera. Ahora con casi 16 años no tiene muy claro en qué le gustaría trabajar pero no tiene expectativas de seguir estudiando.

Por otra parte, el profesorado percibe que las familias y el alumnado sienten un rechazo hacia la escuela. Creen que entienden la educación formal como una imposición de una cultura que no es la propia (Entrevista a docentes X, N e Y; 22 y 30.03.10). Además, opinan que las familias mantienen cierta desconfianza hacia ellos.

Hay un rechazo, las familias hasta que no se dan cuenta de que pueden confiar en los maestros, que ellos quieren a sus hijos y son buenos, no confían en ellos. (Primera entrevista a docente $X, 22.03 .10$ ).

Finalmente, el profesorado también tiene prejuicios hacia las familias y el alumnado. Sobre las familias opinan que no le otorgan valor a la educación académica, más allá de la función asistencial.

En general, se sienten obligados y por eso les traen al colegio, pero que además se impliquen en esa educación para ellos no tiene ningún valor. (Primera entrevista a docente $N$, 30.03.10). 
Este rechazo también se puede observar con la actitud de una parte del profesorado hacia familias y alumnado. Por ejemplo, al tratar de mantener una distancia constante con las familias, incluso en aspectos que marcan la dinámica escolar. Por ejemplo, en el hecho de utilizar puertas distintas para entrar al centro. El profesorado entra por delante y las familias y el alumnado por la puerta de atrás.

A la hora de salir los niños, que salen los niños, los profesores, que es el único momento en que muchos profesores pueden hablar con los padres, pueden tener ese intercambio... ¿Por qué se abre solo la puerta pequeña? (Miembro del equipo directivo. En sesión final de formación, 04.06.10).

El profesorado defiende la participación democrática, pero al mismo tiempo no se sitúa en un plano de horizontalidad con las familias.

Se tendría que dar un paso adelante y abrirse más, tratar de igual a igual, bajar también el discurso. Estar más relajados. (Segunda entrevista a docente $X, 22.03 .10$ ).

También con respecto al alumnado podemos destacar algunas situaciones en las que el profesorado muestra menosprecio. Por ejemplo, un día una maestra se llevó, por descuido, las tijeras de otra maestra, volvió a devolvérselas diciendo: Lo siento, al final se nos «apega» también esto de nuestros alumnos.

Finalmente, el clima de convivencia se caracteriza por numerosos conflictos entre el alumnado, así como con relaciones distantes entre el profesorado y las familias. En primer lugar, no hay un consenso a la hora de establecer las normas, sino que estas han sido impuestas por el profesorado y en algunos casos son incumplidas por ellos mismos. Por ejemplo, el hecho de que no se pueda comer chicle en clase y algún maestro/a lo haga; o la puntualidad en la asistencia al centro (Sesión 2 del taller sobre resolución de conflictos, 05.02.10).

Además, el alumnado manifiesta su desacuerdo respecto a la incongruencia entre las normas establecidas en la escuela y las de su casa. También el estudiantado destaca la falta de coherencia entre los castigos impuestos ante el incumplimiento de las normas (Grupo de discusión del alumnado de tercer ciclo y primer ciclo de la ESO, 20.01.10). Por ejemplo, diferencian entre el tipo de insulto y califican el «cagarse en los muertos» como una falta de respeto muy grave, por eso no están de acuerdo en que este castigo se resuelva quedándose unos días sin patio. 
A la pregunta de si resuelven de la misma manera los conflictos las familias y el profesorado, responden:

No, los profesores lo hacen hablando. Algunas madres, pues lo hacen peleando. (Grupo de discusión alumnado de segundo y tercer ciclo de primaria, 20.01.10).

En este sentido, el alumnado prefiere resolver los conflictos mediante el diálogo, pues dicen que es más efectivo porque pueden llegar a entender por qué se les prohíbe hacer determinadas cosas y de esta manera no ser reincidentes en el incumplimiento de dicha norma (Entrevista a alumna del aula especial del primer ciclo de la ESO, 11.05.10).

Por otra parte, en la escuela tampoco se les aplica el mismo castigo a dos niños que hayan cometido la misma falta, sino que tienen la percepción de que el profesorado se deja llevar por sus preferencias a la hora de imponer los castigos. Además, afirman que no todo el profesorado castiga de la misma manera ante la misma situación. Creen que una parte del profesorado es muy autoritario, mientras otros tratan de reflexionar más con el alumnado e imponen castigos, a su juicio, menos severos.

Además, existe una relación entre los conflictos que aparecen en la escuela y los de la calle. Aunque tanto las familias como el profesorado insisten en la necesidad de no intervenir en aquellos que no sean competencia suya (grupo de discusión de las familias, 20.01.10), en infinidad de situaciones los conflictos trascienden de la familia a la escuela y viceversa. Según comenta el alumnado, en algunos casos las familias intervienen en los conflictos que consideran de la escuela, y los resuelven discutiendo directamente con el alumno que ha ofendido a sus hijos o hijas, o tratando el tema con violencia con la familia del otro niño o niña.

Para evitar dichas situaciones, el profesorado manifiesta la importancia de resolver los conflictos de la escuela dentro de la misma. Pero con normas y consecuencias impuestas, el alumnado no se siente satisfecho y al salir del colegio cuenta su preocupación a su familia.

$N$. Por culpa de otros niños que son muy chivatos los padres y las madres se enganchan. (Grupo de discusión alumnado de segundo ciclo y quinto de Primaria 20.1.10).

Por otra parte, el profesorado mantiene contacto con las familias y les informan sobre conflictos que no saben cómo resolver en el centro. Para algunas de las familias la opinión del profesorado es importante y ante un 
castigo en el colegio llega otro en casa (Entrevista a alumna del aula especial del primer ciclo de la ESO, 11.05.10).

En la calle el comportamiento del alumnado es distinto, median con otras normas y otras consecuencias. Por eso, una misma falta cometida dentro o fuera del colegio conlleva consecuencias distintas.

Si me peleo con alguien en la escuela entonces me encierran con llave o me castigan, pero si es con alguien de la calle, entonces no me dicen nada. (Entrevista a alumna del aula especial del primer ciclo de la ESO, 11.05.10).

Aunque parece que las familias y la escuela sean dos mundos diferentes con el único punto en común que son los hijos/alumnado, tanto para el profesorado como para las familias es importante todo lo que ocurre en los distintos contextos.

\section{CONCLUSIONES}

Como hemos podido observar en los resultados, el silenciamiento del alumnado en esta escuela viene influenciado por una cultura profesionalista del profesorado. Este considera que debe apostar por una mayor participación comunitaria y una democratización de las decisiones en el centro, pero se resiste a abandonar un enfoque profesionalista que le legitima a ser el que tome la última decisión. Su percepción estereotipada de la diversidad cultural y de lo que puede esperar del contexto en el que trabaja, dificulta su posicionamiento inclusivo e intercultural a la hora de cambiar su mirada sobre la escuela.

En este posicionamiento docente, el papel protagonista que el profesorado ocupa en el centro condiciona de manera decisiva su relación con el alumnado. Como apunta Guerrero (2007), «El poder del profesor se hace sentir de manera protagonista en la relación pedagógica; bien sea como actividad de imposición e inculcación del arbitrario cultural, en las pedagogías visibles o pasivas; o bien a partir de la posición de ventaja que posee en la negociación de la transmisión cultural en las pedagogías invisibles o activas» (p. 56). Relación que, como describe Guerrero, viene reforzada por la propia institución escolar en la que las características específicas de los espacios de enseñanza formal junto con el uso de un lenguaje docente difícil y alejado de los intereses de la mayoría del grupo clase, provoca un mayor distanciamiento e invisibilización del alumnado.

Para transformar dicha cultura hacia un modelo de participación más democrática y basada en la validez del argumento de todos los miembros, es 
necesario transformar también el liderazgo, puesto que en la construcción de una escuela abierta a la comunidad, este no puede recaer únicamente en manos del profesorado, sino que para avanzar hacia un modelo pedagógico basado en la participación es necesario un liderazgo compartido. Para ello, en muchas ocasiones necesitaremos «romper la seguridad tradicional de las relaciones de poder que se establecen entre profesores y alumnos y redefinir las fronteras de la posibilidad» (Rudduck y Fielding, 2006: 225). Como dice Fielding (2004, p. 296):

La transformación requiere una ruptura con lo tradicional, para lo cual se necesita tanto del profesorado como del alumnado. De hecho, requiere una transformación del significado de ser estudiante; del significado de ser profesor. En realidad, requiere de la mezcla e interdependencia de ambos.

En este sentido, entendemos que la voz del alumnado es importante y está directamente comprometida con el cambio hacia la inclusión y la democracia participativa en los centros (Traver y otros, 2010; Fielding, 2011; Rudduck y Flutter, 2007; Thompson, 2007, Susinos, 2013). Además, es necesario el uso de estructuras colaborativas, en las que estudiantes y profesionales trabajen juntos (Messiou, 2013). Cuando esta dinámica entra a formar parte de la cultura de la escuela, algunos autores lo identifican como un progreso hacia lo que se define como una cultura inclusiva (Dyson, Howes y Roberts, 2004). Pero, como comentan Calvo, Rodríguez-Hoyos y García (2012), a la hora de trabajar la participación de los estudiantes encontraremos dificultades por la ausencia de una cultura democrática en los centros, que por el contrario, se irá construyendo en la medida en que cambie tanto el rol del alumnado como del profesorado. «El alumnado necesitará incorporarse al proyecto en un rol poco conocido para él» (Susinos, 2013, p. 129). En este punto, es importante destacar que la participación del alumnado no se dirige únicamente a la de unos pocos, sino a la de todos y todas. De manera que la voz del alumnado estará más presente, en la medida en que participe más y activamente todo el alumnado en la toma de decisiones sobre distintos aspectos del centro y de su funcionamiento (Susinos y Ceballos, 2012).

Finalmente, hablar de la voz del alumnado nos permite visibilizar y convertir en un "problema pedagógico» lo que con frecuencia pasa inadvertido (Susinos, 2013). Este aspecto se ha podido observar en el estudio, cuando el alumnado ha opinado sobre aquellas cuestiones que le preocupaban o le interesaban y ha mostrado algunas de las incongruencias del centro. Por ejemplo, cuando el estudiantado ha manifestado su incomprensión y desacuerdo sobre el incumplimiento de normas que el propio profesorado ha impuesto. 
Así pues, hablar de la voz del alumnado pone en el centro de las preocupaciones de las escuelas y de los profesores, la inquietud de cómo es posible organizarse para que la participación de los alumnos sea auténtica y sea en sí misma un objetivo pedagógico relevante (Susinos, 2012, p. 17-18).

Para concluir, destacar que en el estudio se evidencia lo complejo de un proceso de transformación y lo importante que es la reflexión sobre la propia práctica, para tomar conciencia de las propias contradicciones. Poner en primer plano la voz del alumnado y su papel en la construcción de una escuela más participativa y democrática, nos permite profundizar en las dificultades que supone la transformación de la cultura escolar y detectar los factores que generan una resistencia al cambio.

Ha sido precisamente la perspectiva del alumnado la que ha ayudado a poner sobre la mesa las posibles contradicciones y dilemas que supone este proceso de cambio escolar.

\section{NOTAS}

1 Centros con un $33 \%$ de alumando en riesgo de exclusión social, con recursos personales y materiales extra.

2 MEICRI (Grupo de investigación de la Universitat Jaume I. Mejora educativa y ciudadanía crítica, http://meicri.uji.es/) 


\section{REFERENCIAS BIBLIOGRÁFICAS}

Ainscow, M. (2002). Rutas para el desarrollo de prácticas inclusivas en los sistemas educativos. Revista de educación, 327, 69-82.

Becker, H. S. (1953). The teacher in the authority system of the public school. Journal of the Educational Sociology, 27, 128-141.

Bucknall, S. (2009). Children as researchers: exploring issues and barriers in English primary schools. [PhD thesis]. The Open University.

Calvo, A., Rodríguez-Hoyos, C., y García, M. (2012). Lo mejor de todo es que nos escucháis. Investigar el aumento de la participación de los estudiantes en los programas de diversificación y de cualificación profesional inicial. Revista de Educación, 359, 164-183.

Cohen, L. y Manion, L. (1990). Métodos de investigación educativa. Madrid: La Muralla.

Dyson, A., Howes, A. \& Roberts, B. (2004). What do we really know about inclusive schools? A systematic review of the research evidence. En D. Mitchell (Ed.), Special Educational Needs and Inclusive Education: Major Themes in Education. London: Routledge.

Echeíta, G. (2011). El proceso de inclusión educativa en España. ¡Quien bien te quiere te hará llorar! CEE Participación Educativa, 18, 117-128.

Fielding, M. (2004). Transformative approaches to student voice: Theoretical underpinnings, recalcitrant realities. British Educational Research Journal, 30(2), 295-311.

Fielding, M. (2011). La voz del alumnado y la inclusión educativa: una aproximación democrática radical para el aprendizaje intergeneracional. Revista Interuniversitaria de Formación del Profesorado, 70(25), 31-61.

Gale, T. y Densmor, K. (2007). La implicación del profesorado. Una agenda de democracia radical para la escuela. Barcelona: Octaedro.

Glaser, B. G. y Strauss, A. L. (1967). The discovery of Grounded Theory. Chicago (EUA): Aldine.

Guerrero, A. (2007). El análisis sociológico del profesorado: categoría social y agente educativo. Educación y Futuro, 17, 43-70.

Hargreaves, A. y Fink, D. (2008). El Liderazgo Sostenible. Siete principios para el liderazgo en centros educativos innovadores. Madrid: Morata.

Imbernon, F. (2007). La formación y el desarrollo profesional del profesorado. Hacia una nueva cultura profesional. Barcelona: Graó. 7. a edición.

Marcelo, C. y Estebaranz, A. (1999). Cultura escolar y cultura profesional: los dilemas del cambio. Educar, 24, 47-69.

Márquez, C. y Sandoval, M. (2016). ¿Para cuándo la mejora de participación de los estudiantes en los centros educativos? Intersticios: Revista Sociológica de Pensamiento Crítico, 10 (2), 21-33.

Messiou, K. (2013). El compromiso con la voz del alumnado: uso de un marco de trabajo para abordar la marginación en las escuelas. Revista de Investigación en Educación, 11(3), 97-108.

Rojas, S.; Haya, I. y Lázaro, S. (2012). La voz del alumnado en la mejora escolar: niños y niñas como investigadores en Educación Primaria, Revista de Educación, 359, 81-101. 
Rudduck, J. \& Fielding, M. (2006). Students voice and the perils of popularity. Education Review, 58 (2), 219-231.

Rudduck, J y Flutter, J. (2007). Cómo mejorar tu centro escolar dando la voz al alumnado. Madrid: Morata.

Rubin, H. \& Rubin, I. (1995). Qualitative Interviewing: The Art of Hearing Data. Thousand Oaks, CA: Sage.

Santos Guerra, M. A. (2010). Una pretensión problemática: educar para los valores y preparar para la vida. Revista de Educación, 351, 23-47.

Stake, R. (2007). Investigación con estudio de casos. Madrid: Ediciones Morata.

Susinos, T. (2012). Las posibilidades de la voz del alumnado para el cambio y la mejora educativa. Revista de Educación, 359, 16-23.
Susinos, T. y Ceballos, N. (2012). Voz del alumnado y presencia participativa en la vida escolar. Apuntes para una cartografía de la voz del alumnado en la mejora educativa. Revista de Educación, 359, 24-44.

Susinos, T. (2013). Desde el mismo lugar no vemos lo mismo. Investigar la participación de los estudiantes como un proceso multivocal. Revista de Investigación en Educación, 11(3), 120-132.

Traver, J. A., Sales, A. y Moliner, O. (2010). Ampliando el territorio: algunas claves sobre la participación de la comunidad educativa. REICE. Revista Iberoamericana sobre calidad, eficiencia y cambio en educación, 8(3), 96-119.

Waller, W. (1932). The Sociology of teaching. New York: Wiley. 


\section{PERFIL ACADÉMICO Y PROFESIONAL DE LOS AUTORES}

Paula Escobedo Peiro. Licenciada en Psicopedagogía y doctora en Educación por la Universitat Jaume I de Castellón, con premio extraordinario de Licenciatura. Es profesora ayudante doctor tipo II, en el Departamento de Educación, Universitat Jaume I. Ha realizado trabajos de investigación sobre la escuela intercultural inclusiva y la cultura escolar. Sus líneas de investigación actuales son la escuela intercultural inclusiva, las prácticas de aula inclusivas, las emociones en la educación y las historias de vida.

Auxiliadora Sales Ciges. Licenciada y doctora en Filosofía y Ciencias de la Educación (sección CC. de la Educación) por la Universidad de Valencia, con premios extraordinarios de Licenciatura y de Doctorado. Profesora Titular del Departamento de Educación de la Universitat Jaume I. Centra su línea de investigación en la educación intercultural e inclusiva, participando en distintos proyectos investigadores tanto de ámbito local como autonómico.

Joan Traver Martí. Doctor en Educación y Licenciado en Filosofía y CCEE. Es profesor Titular del Departamento de Educación de la Universitat Jaume I de Castellón. Ha realizado trabajos de investigación sobre el aprendizaje cooperativo y la enseñanza de valores y actitudes y tiene algunas publicaciones sobre esta temática. Sus líneas de investigación actuales son el aprendizaje cooperativo, la escuela intercultural inclusiva, la participación socio-comunitaria.

Dirección de los autores: Av. de Vicent Sos Baynat, s/n

12071 Castelló de la Plana

E-mail: paula.escobedo@uji.es

asales@uji.es

jtraver@uji.es

Fecha Recepción del Artículo: 05. Marzo. 2014

Fecha modificación Artículo: 23. Julio. 2014

Fecha Aceptación del Artículo: 25. Enero. 2015

Fecha Revisión para Publicación: 13. Marzo. 2017 